\title{
Correlation Analysis of Thyroid Function and Autoantibodies in Graves' Disease Patients with Different lodine Nutritional Status
}

\author{
Jing Feng ${ }^{1}$, Cuicui Wang ${ }^{2}$, Zhaoxin $\mathrm{Mu}^{2}$, Xinsheng $\mathrm{Li}^{1}$, Zhenjiang $\mathrm{Hou}^{2}{ }^{2}$ * \\ ${ }^{1}$ Cangzhou Central Hospital, Cangzhou, China \\ ${ }^{2}$ Institute of Thyroid Diseases Affiliated to Cangzhou Medical College, Cangzhou Thyroid Disease Engineering Technology Research Center, \\ Cangzhou, China
}

Email address:

houzhenjiang@sina.com (Zhenjiang Hou)

${ }^{*}$ Corresponding author

\section{To cite this article:}

Jing Feng, Cuicui Wang, Zhaoxin Mu, Xinsheng Li, Zhenjiang Hou. Correlation Analysis of Thyroid Function and Autoantibodies in Graves' Disease Patients with Different Iodine Nutritional Status. Science Journal of Public Health. Vol. 8, No. 6, 2020, pp. 168-176.

doi: 10.11648/j.sjph.20200806.13

Received: September 23, 2020; Accepted: October 30, 2020; Published: November 27, 2020

\begin{abstract}
Objective: To investigate the correlation between thyroid function stratification and autoantibody titers in patients with Graves' disease (GD) under different iodine nutrition conditions. Methods: The levels of serum thyroid hormones, autoantibodies and urinary iodine in 100 patients with different thyroid function GD [GD-A, GD-B] and 60 healthy subjects are detected by electrochemical immunoluminescence and iodine-catalyzed arsenic-cerium method. Results: The proportion of GD patients with the sum of iodine overdosage and iodine overdosage group is consistent with iodine adequate group, which is significantly higher than iodine deficiency group, and the (Median Urine Iodine, MUI) of GD-A group is significantly higher than GD-B group. Urinary iodine levels in GD group and GD-A group are positively correlated with serum FT3 and FT4 $(\mathrm{P}<0.05)$, and negatively correlated with (thyroid stimulating hormone, $\mathrm{TSH})(\mathrm{P}<0.05)$. The levels of serum (thyroid peroxidase antibody, TPOAb) and (thyroid globulin antibody, TGAb) in each GD group are significantly higher than those in the control group. Serum TSH levels in the high TPOAb group and high TgAb group are lower than those in the low TPOAb group, low $\mathrm{TgAb}$ group and control group, respectively. The levels of FT3 and FT4 are higher than those of the low TPOAb group and the low TgAb group, however, there is no significant difference between the FT3 of low TgAb and T3 and T4 levels that belong to the control group, high TPOAb group, high TgAb group, low TPOAb group, and low TgAb group. In conclusion: GD patients with different thyroid functions have corresponding changes in their thyroid hormone and autoantibody levels under different iodine nutrition conditions, which indicates that iodine nutrition status involve in and play an important role in the development of GD with different thyroid functions and different antibody levels.
\end{abstract}

Keywords: Thyroid Hormone, Median Urine Iodine, Thyroid Autoantibodies, Graves’ Disease, Hyperthyroidism

\section{Introduction}

Graves' Disease (GD) that also known as toxic diffuse goiter, is a common clinical endocrine disease and the most common autoimmune thyroid disease (Autoimmune thyroid disease, AITD), which is the highest incidence of all types of hyperthyroidism. About $80 \%$ to $86 \%$ of hyperthyroidism is caused by GD. The clinical symptoms or signs of GD mainly include diffuse symmetrical goiter and hyperfunction, exophthalmos, and hypermetabolic state. It can be onset at any age, but it is more common in women. The adverse effects of this disease on multiple systems are very prominent, which can lead to multiple organ damage [1], poor treatment effects, and serious sequelae. In recent years, the incidence rate has been increasing, which seriously affects the quality of life of patients [2]. There are many factors in the pathogenesis of GD, such as environmental, genetic, and autoimmune dysfunction, all that can lead to specific autoimmune reactions in the thyroid gland, diffuse goiter, and hyperthyroidism [3]. Clinical researches have 
shown that there are many reasons for the increase in the incidence of GD, and iodine nutritional status may be one of the influencing factors. The effect of iodine on GD has received extensive clinical attention. Researches have shown that excessive iodine intake increases the incidence of GD and the positive rate of thyroid autoantibodies. As the disease worsens, the recurrence rate also increases [4]. A large number of epidemiological investigations have been conducted on the relationship between urinary iodine (UI) and hyperthyroidism and GD, but the results of the research are not the same. Clinical researches have been carried out on the thyroid function and autoantibody levels of GD patients, but GD is the research object. There are few studies on the thyroid function and autoantibody levels of GD patients with iodine nutrition level. In particular, there are few reports on the correlation between thyroid function stratification and antibody titer in GD patients with different iodine nutrition status. The author carried out this research to explore the changes of iodine nutritional status in the pathogenesis of GD patients with different thyroid function and autoantibody levels, and to provide scientific basis for the development of precise treatment and prevention of GD and its pathogenesis.

\section{Materials and Methods}

\subsection{Research Objects and Groups}

A total of 100 untreated inpatients with GD who are in the endocrinology department of Cangzhou Central Hospital and Cangzhou People's Hospital from September 2019 to August 2020 are selected as the experimental group, including 24

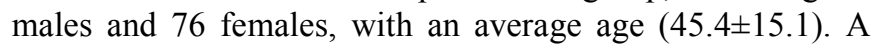
total of 60 healthy subjects with matching gender and age are selected as the control group (NC group), including 16 males and 44 females, with an average age of $(44.8 \pm 16.9)$ years. There are no statistically significant differences in gender and age in each group. All the patients met GD diagnostic criteria in Chinese Thyroid Disease Diagnosis and Treatment Guidelines formulated by Chinese Society of Endocrinology, Chinese Medical Association in 2008 [5]. According to the results of thyroid hormone examination and whether there are corresponding clinical symptoms or signs, they are divided into GD-A group (clinical hyperthyroidism: with symptoms or signs of hyperthyroidism, while FT3 $>7.1 \mathrm{pmol} / \mathrm{L}$, FT4 $>22 \mathrm{pmol} / \mathrm{L}, \quad \mathrm{TSH}<0.27 \mathrm{mU} / \mathrm{L}$ ) and GD-B group (subclinical hyperthyroidism: no symptoms or signs of hyperthyroidism, FT3 and FT4 are normal, TSH $<0.27 \mathrm{mU} / \mathrm{L}$ ). Exclusion criteria: (1) Complicated with other autoimmune system diseases; (2) Acute and chronic infections, malignant tumors, pregnancy and allergic diseases; (3) Past history of diabetes, abnormal liver function and blood system diseases; (4) Those who have used glucocorticoid therapy in the past 30 days. The evaluation and analysis of iodine nutritional status adopt the standards issued by the World Health Organization (WHO), the United Nations Children's Fund (UNICEF) and the International Council for the Control of Iodine Deficiency
Disorders (ICCIDD) in 2007. Median urinary iodine (MUI) $<100 \mu \mathrm{g} / \mathrm{L}$ is iodine deficiency, $100-199 \mu \mathrm{g} / \mathrm{L}$ is the right amount of iodine, $200-299 \mu \mathrm{g} / \mathrm{L}$ is iodine excess, $\geq 300 \mu \mathrm{g} / \mathrm{L}$ is iodine excess [6]. This research is approved by the ethics committee, and the research subjects sign an informed consent form.

\subsection{Research Methods and Projects}

\subsubsection{Specimen Collection}

All subjects are fasted for more than 12 hours, and collected $10-20 \mathrm{ml}$ of fasting mid-section urine in the morning, placed in a clean polyethylene plastic tube, stored in a refrigerator, and collected fasting venous blood at 8:00-10:00 5mL, after standing at room temperature for $1 \mathrm{~h}$, centrifuge at $3000 \mathrm{r} / \mathrm{min}$ for $10 \mathrm{~min}$, take serum, determine thyroid hormone content and autoantibody level. Avoid eating kelp, seaweed and other high-iodine foods and iodine-containing medicines, and avoid increasing drinking water for the first 1 day.

\subsubsection{Project Detection}

(1) Use Cobas E601 immuno-chemiluminescence instru-ment from Roche Co., Ltd. to determine serum TSH, total triiodothyronine (TT3), total thyroxine (TT4), free triiodothyronine (FT3), free thyroxine (FT4), TPOAb, TGAb, the reagents are all original Roche reagents. Due to regional differences and the influence of equipment, reagents and other factors, the reference value ranges of each hospital are different. The normal reference intervals for thyroid hormones and autoantibodies in this hospital are as follows: TSH 0.27 4.2 $\mathrm{mIU} / \mathrm{L}$, FT3 2.8 7.1 pmol/L, FT4 12 22 pmol/L, T3 $1.3 \sim 3.10 \mathrm{nmol} / \mathrm{L}, \mathrm{T} 466 \sim 181 \mathrm{nmol} / \mathrm{L})$, TPOAb $0 \sim 34 \mathrm{IU} / \mathrm{ml}$, $\mathrm{TgAb} 0 \sim 115 \mathrm{IU} / \mathrm{ml}$. (2) Urine iodine determination adopts the principle of iodine-catalyzed arsenic-cerium reaction [7], and the urinary iodine content is detected by arsenic-cerium catalytic spectrophotometry (WS/T107-2006). The kit is produced by Wuhan Zhongsheng Biochemical Technology Co., Ltd., and the instrument is made by Hitachi, Japan Model 7600-110 automatic biochemical analyzer.(3) Check the thyroid morphology with color Doppler ultrasound. The main manifestations of HT patients are diffuse enlargement of the thyroid gland, thickened light spots, uneven distribution, and diffuse hypoechoic.

\subsubsection{Statistical Methods}

Statistical analysis is carried out using SPSS 20.0 statistical software. Counting data is expressed as rate (\%), and the comparison is made by $\chi^{2}$ test. Normal distribution measurement data is expressed as mean \pm standard deviation ( $\bar{x} \pm \mathrm{s}$ ), using the $\mathrm{t}$ test, with $\mathrm{P}<0.05$, which indicates that the difference is statistically significant. Non-normally distributed measurement data are described by median. Kruskal-Wallis $\mathrm{H}$ test is used for comparison among multiple groups, and Spearman correlation analysis is used for univariate correlation analysis. Pearson or Spearman correlation analysis is used for Pearson or Spearman correlation analysis. $\mathrm{P}<0.05$ is considered statistically significant. 


\section{Results}

\subsection{Comparison of Clinical Characteristics of GD Patients with Different Thyroid Functions}

The gender and age of $100 \mathrm{GD}$ patients with different thyroid function are compared with the control group, and the difference is not statistically significant $\left(\chi^{2}=2.681, \mathrm{P}=0.536\right.$ vs $\left.\chi^{2}=0.873, \mathrm{P}=0.627\right)$. Therefore, the research objects are comparable. See Table 1.

Table 1. Comparison of gender and age of HT patients in each group

\begin{tabular}{llll}
\hline Group & Number of cases & Gende (male, $\mathbf{n}, \mathbf{\%})$ & Age (year old, $\overline{\boldsymbol{x}} \pm \mathbf{s})$ \\
\hline GD & 100 & $24(24.00)$ & $(46.1 \pm 17.3)$ \\
GD-A & 81 & $21(25.93)$ & $(45.3 \pm 17.9)$ \\
GD-B & 19 & $5(26.32)$ & $(43.6 \pm 16.7)$ \\
NC & 60 & $16(26.67)$ & $(44.9 \pm 18.2)$ \\
$\chi^{2}$ & & 2.681 & 0.873 \\
$P$ & & 0.536 & 0.627 \\
\hline
\end{tabular}

\subsection{Comparison of Thyroid Hormones and Autoantibodies in GD Patients with Different Iodine Nutritional Status}

According to the urine iodine level, the thyroid hormone and autoantibody levels of $100 \mathrm{GD}$ patients with different iodine nutritional status are compared. The results of the iodine deficiency, adequate iodine, iodine beyond and iodine excess groups are 92.31, 171.28, 242.63 and $311.46 \mu \mathrm{g} / \mathrm{L}$, respectively. The composition ratio of GD patients with different urinary iodine concentrations, the sum of iodine overdose and iodine overdose group is consistent with the proportion of iodine adequate group, which is significantly higher than iodine deficiency group. The MUI in the GD-A group is positively correlated with serum FT3 and FT4 $(\mathrm{r}=0.316, \mathrm{P}=0.031 ; \mathrm{r}=0.328, \mathrm{P}=0.036$; the MUI in the GD-B group is positively correlated with serum FT3 and FT4 $(\mathrm{r}=0.289, \mathrm{P}=0.037 ; \mathrm{r}=0.310, \mathrm{P}=0.0352)$, which is negatively correlated with TSH $(\mathrm{r}=-0.324, \mathrm{P}=0.034)$. In the iodine deficiency group, the TSH level is higher than the other three groups. With the increase of urinary iodine level, the serum TPOAb and TGAb levels of patients in the GD-A and GD-B groups also increase. See Table 2.

Table 2. Comparison of thyroid function and serum TPO-Ab and TG-Ab levels in GD patients with different urine iodine concentrationsof Cases.

\begin{tabular}{|c|c|c|c|c|c|c|c|c|}
\hline Group & Numbe & TSH (mIU/L) & FT3 (pmol/L) & FT4 (pmol/L) & T3 (nmol/L) & T4 (nmol/L) & TPOAb (IU/ml) & TGAb (IU/ml) \\
\hline Deficienc & 11 & $0.24 \pm 0.021$ & $16.48 \pm 4.02$ & $42.51 \pm 11.3$ & $1.78 \pm 0.74$ & $148.8 \pm 20.7$ & $264.7 \pm 192.4$ & $182.6 \pm 37.41$ \\
\hline Right amount & 43 & $0.23 \pm 0.026$ & $24.37 \pm 7.47$ & $51.43 \pm 10.6$ & $1.69 \pm 0.70$ & $156.2 \pm 21.9$ & $316.4 \pm 186.9$ & $218.7 \pm 39.25$ \\
\hline Beyond & 31 & $0.21 \pm 0.028$ & $38.06 \pm 8.31$ & $59.32 \pm 11.8$ & $1.81 \pm 0.73$ & $154.7 \pm 23.6$ & $376.5 \pm 193.2$ & $236.4 \pm 41.28$ \\
\hline Excess & 15 & $0.19 \pm 0.023$ & $41.32 \pm 10.1$ & $67.16 \pm 12.3$ & $1.72 \pm 0.69$ & $161.3 \pm 21.4$ & $482.6 \pm 190.7$ & $276.3 \pm 46.19$ \\
\hline
\end{tabular}

\subsection{Comparison of Thyroid Hormone and Autoantibody Levels in GD Patients with Different Thyroid Functions}

100 cases of GD patients and GD-A, GD-B group thyroid hormone and autoantibody levels are compared, the results show that the serum TSH levels of the GD group, the GD-A group, and the GD-B group are significantly lower than the NC $(\mathrm{P}<0.05)$. There is a statistically significant difference between GD-A and GD-B groups $(\mathrm{P}<0.05)$. With the hyperthyroidism of GD-B and GD-A, the TSH level gradually decrease $(\mathrm{P}<0.05)$. Serum FT3 and FT4 levels in GD, GD-A and GD-B groups are higher than $\mathrm{NC}$, but the difference between GD-B and NC groups is not statistically significant $(\mathrm{P}>0.05)$. There is no significant difference in serum $\mathrm{T} 3$ and T4 levels among the GD, GD-A and GD-B groups and the NC group $(\mathrm{P}>0.05)$. Serum TPOAb and TGAb levels in GD, GD-A and GD-B groups are significantly higher than NC $(\mathrm{P}<0.01)$, and serum TPOAb and TGAb levels in GD-A are also higher than those in GD-B group. See Table 3.

Table 3. Comparison of GD thyroid hormone and autoantibody levels with different thyroid functions.

\begin{tabular}{|c|c|c|c|c|c|c|c|c|}
\hline Group & Cases & TSH (mIU/L) & FT3 (pmol/L) & FT4 (pmol/L) & T3 (nmol/L) & T4 (nmol/L) & TPOAb IU/ml & TGAb IU/ml \\
\hline G D & 100 & $0.12 \pm 0.03 *$ & $34.52 \pm 5.81 *$ & $52.51 \pm 10.9 *$ & $1.81 \pm 0.76$ & $108.49 \pm 20.16$ & $386.5 \pm 184.9 * \mathbf{\Lambda}$ & $247.13 \pm 70.2^{* \mathbf{\Delta}}$ \\
\hline GD-A & 81 & $0.09 \pm 0.06^{*}$ & $42.17 \pm 6.82 *$ & $64.13 \pm 12.4^{*}$ & $1.91 \pm 0.69$ & $110.32 \pm 18.75$ & $408.9 \pm 173.2 * \Delta$ & $269.27 \pm 74.1 * \boldsymbol{\Lambda}$ \\
\hline GD-B & 19 & $0.15 \pm 0.04 *$ & $5.35 \pm 0.62$ & $21.02 \pm 8.02$ & $1.79 \pm 0.82$ & $101.96 \pm 20.91$ & $359.2 \pm 179.6^{* \boldsymbol{\Delta}}$ & $229.30 \pm 67.9^{* \boldsymbol{\Lambda}}$ \\
\hline
\end{tabular}

Note: Compared with the control group, * is $\mathrm{P}<0.05, * \boldsymbol{\Delta}$ is $\mathrm{P}<0.01$.

\subsection{Comparison of Thyroid Hormone Levels in HT Patients with Different TPO-Ab and TG-Ab Levels}

The number of 100 GD patients with positive TPOAb and TGAb antibodies are 82 and 63 , respectively. The median antibody levels are $361.4 \mathrm{IU} / \mathrm{mL}$ and $238.6 \mathrm{IU} / \mathrm{mL}$, respectively. Using this as a cut-off value, GD is divided into high TPOAb group, low TPOAb group, high TGAb group, low TGAb group, comparison of thyroid hormone levels in GD patients with different TPOAb and TGAb levels. The results show that the TSH levels of the four groups of high and low TPOAb and TGAb of GD patients are significantly lower than the NC group, and the levels of FT3 and FT4 are significantly higher 
than the NC group, and the differences are statistically significant $(\mathrm{P}<0.05)$. And the serum TSH levels of the high TPOAb group and the high TGAb group are lower than the low TPOAb group, the low TGAb group and the control group, while the FT3 and FT4 levels are higher than the low TPOAb group and the low TGAb group, respectively. The differences are statistically significant $(\mathrm{P}<0.05)$. However, there is no statistically significant difference in T3, T4 levels and $\mathrm{NC}$ in the high TPOAb group, high TGAb group, low TPOAb group, and low TGAb group. See Table 4.

Table 4. Comparison of thyroid hormone levels in GD patients with different TPOAb and TGAb levels.

\begin{tabular}{lllllll}
\hline Group & n & TSH (mIU/L) & FT3 (pmol/L) & FT4 (pmol/L) & T3 (nmol/L) & T4 (nmol/L) \\
\hline High TPOAb & 45 & $0.14 \pm 0.02^{*}$ & $39.32 \pm 1.31^{*}$ & $63.42 \pm 3.28^{*}$ & $1.78 \pm 0.69$ \\
Low TPOAb & 37 & $0.16 \pm 0.03^{*}$ & $31.41 \pm 1.43^{*}$ & $51.37 \pm 4.12^{*}$ & $1.80 \pm 0.58$ \\
High-TGAb & 34 & $0.13 \pm 0.03^{*}$ & $42.47 \pm 1.26^{*}$ & $54.28 \pm 3.62^{*}$ & $1.81 \pm 0.46$ \\
Low-TGAb & 29 & $0.15 \pm 0.05^{*}$ & $29.72 \pm 1.54^{*}$ & $38.61 \pm 3.96^{*}$ & $1.75 \pm 0.63$ \\
NC & 60 & $0.26 \pm 0.02$ & $4.91 \pm 0.58$ & $19.14 \pm 7.09$ & $1.03 .6 \pm 22.4$ & $1.82 \pm 0.42$ \\
\hline
\end{tabular}

Note: Compared with the control group, * means $\mathrm{P}<0.05$.

\section{Discussion}

Thyroid disease is a global disease. According to statistics from WHO in 2000, there are at least 130 countries and regions in the world with iodine deficiency, affecting 2.225 billion people. In 1990, the United Nations summit passed a declaration calling for the elimination of iodine deficiency disorders (IDD) globally by 2000 . Since the implementation of universal salt iodization (USI). However, with the progress of USI, the incidence of various thyroid diseases is on the rise. That the urine iodine level $(306.9 \pm 116.2 \mu \mathrm{g} / \mathrm{L})$ of 196 patients with thyroid disease is significantly higher than the control group $(195.7 \pm 101.1 \mu \mathrm{g} / \mathrm{L}, \mathrm{P}<0.01)$, which indicates that iodine excess is positively correlated with thyroid disease [8]. The relationship between urinary iodine level and thyroid disease in 160 newly diagnosed patients with thyroid disease in Xiaogan City, Hubei Province, and finds that the MUI and average values are in the hyperfunction group (268.0 and $272.1 \pm 18.8 \mu \mathrm{g} / \mathrm{L})$, normal function group (199.7 and $203.7 \pm 16.2 \mu \mathrm{g} / \mathrm{L})$ and hypofunction group (177.6 and $185.6 \pm 19.3 \mu \mathrm{g} / \mathrm{L})$, the higher the urine iodine level, the higher the thyroid function [9]. That urinary iodine levels in the subclinical hyperthyroidism group, hyperthyroidism group, control group, clinical hypothyroidism group, and subclinical hypothyroidism group increase sequentially [10]. It suggests that iodine nutrition is related to thyroid function. Subclinical thyroid dysfunction is higher than clinical thyroid dysfunction in people with abnormal dysfunction. In particular, the level of urinary iodine in subclinical hypothyroidism is the highest. The proportion of iodine deficiency and iodine excess in clinical and subclinical hypothyroidism is higher, and the nutritional composition ratio of iodine is quite different. The abnormal rate of thyroid function in the urine iodine $\geq 300 \mu \mathrm{g} / \mathrm{L}$ and $<100 \mu \mathrm{g} / \mathrm{L}$ group is higher than $100-199 \mu \mathrm{g} / \mathrm{L}(\mathrm{P}<0.05)$, which suggests that both iodine excess and iodine deficiency can increase the abnormal thyroid function rate. Although the results of research on the relationship between MUI and thyroid function are not the same, the view that iodine intake having an important influence on the spectrum of thyroid disease [11] is consistent.

A large number of epidemiological studies have shown that excessive iodine intake can lead to an increase in the incidence and prevalence of thyroid diseases such as hyperthyroidism and hypothyroidism [12]. Increased iodine intake is an independent risk factor for the onset of hyperthyroidism, and GD accounts for more than $80 \%$ of hyperthyroidism. Excessive intake of iodine, the raw material for the synthesis of thyroid hormones, is prone to hyperthyroidism due to hormone synthesis and excessive secretion for sensitive individuals. Regarding hyperthyroidism caused by excessive iodine, it mainly occurs after iodine supplementation in iodine-deficient areas. Long-term intake of excessive iodine, the body cannot effectively regulate, may lead to hyperthyroidism (iodine induced hyperthyroidism, IIH), hypothyroidism, autoimmune thyroid disease (AITD) and other diseases. AITD is an organ-specific autoimmune disease, mainly including GD disease and HT. Its typical characteristics are thyroid autoantibody positive and abnormal thyroid function [12]. The international thyroid science community generally believes that excess iodine are the main factors that cause hyperthyroidism and AITD. Research data at home and abroad [13] shows that the incidence of hyperthyroidism increase after USI. In 1823, Coindet first reported that hyperthyroidism caused by excessive iodine was caused by the consumption of kelp in iodine-deficient areas. Slowinska-Kleneka and others counted the data of 350,000 thyroid disease patients who underwent fine needle aspiration in Poland in 15 years and found that the incidence of AITD after USI increased from $1.5 \%$ to $5.7 \%$. The WHO and ICCIDD surveys show that the incidence of hyperthyroidism and hyperiodine-related hyperthyroidism after the implementation of USI in Zimbabwe and Australia has increased. The incidence of hyperthyroidism increases significantly after the implementation of USI in Shanghai and Fuzhou. The daily iodine intake of Hangzhou residents has risen from $70 \mu \mathrm{g}$ to $220-330 \mu \mathrm{g}$, which is an over-sufficient iodine nutrition condition. The incidence of hyperthyroidism has risen from $0.9 \%$ to $1.47 \%$. The prevalence of endemic goiter in Wuyishan City after USI 10 years has been reduced from $24.51 \%$ to $11.58 \%(\mathrm{P}<0.01)$, while the prevalence of hyperthyroidism increases from $0.57 \%$ to $1.09 \%(\mathrm{P}<0.01)$. USI significantly reduce the prevalence of endemic goiter, but increase the prevalence of hyperthyroidism [14]. This may be partly related to increased iodine intake. The incidence of hyperthyroidism after iodine supplementation in Dalian non-iodine-deficient areas increase from 10.6/100,000 
people/year to $13.7 / 100,000$ people/year, but the difference is not statistically significant. The incidence of hyperthyroidism in Jilin Province increases from 9.84/100,000 people/year in 1985 to $72.45 / 100,000$ people/year in $2001(\mathrm{P}<0.001)$. Before 1995, there is less iodine, and the incidence of hyperthyroidism does not increase significantly. After 1995, the iodized salt concentration reaches a new level, and the incidence of hyperthyroidism increase significantly [15]. Epidemiological survey data shows that after the implementation of USI, the incidence of clinical hyperthyroidism has increased, and it mainly occurs in iodine-deficient areas [16]. Excessive iodine intake leads to excessive synthesis of thyroid hormones, which leads to hyperthyroidism [12]. Therefore, increased iodine intake is an independent risk factor for the occurrence of hyperthyroidism. In some countries with high iodine intake, such as Japan and the United States, the incidence of GD is significantly higher than countries with sufficient iodine and iodine deficiency. A multi-center study in Austria finds that after implementing USI in iodine-deficient areas, GD and subclinical GD lift the incidence [17]. Yang Fan finds that the prevalence of Garves disease is $1 \%-2 \%$, according to data from the Third National Nutrition Survey of the United States. Conduct an epidemiological survey of Graves' disease in 100,000 people in Daqing area. Logistic regression analysis shows that the increase in iodine intake is closely related to the occurrence of GD and is an independent risk factor. In 2010, a total of 15008 residents aged $\geq 15$ years are sampled in 10 cities in my country. The diagnosis cut-point is $\mathrm{TSH}<0.27 \mathrm{mIU} / \mathrm{L}$, and the prevalence of thyroid diseases is investigated [18]. The results are hyperthyroidism and subclinical hyperthyroidism. The prevalence rates of schizophrenia and Graves' disease are $0.89 \%, 0.72 \%$ and $0.61 \%$, respectively [19]. Investigated the members of $56 \mathrm{GD}$ prone families in 10 cities and counties in Liaoning Province, and study the influence of iodine intake on the incidence of GD, and found that in the GD prone families, the cause of abnormal thyroid function is AITD in their first-degree relatives. The positive rate of autoantibody is $68.6 \%$. There is no significant difference in the incidence of GD among groups with urinary iodine below $500 \mu \mathrm{g} / \mathrm{L}$. The incidence of GD with urinary iodine at $500-599 \mu \mathrm{g} / \mathrm{L}$ is significantly higher than groups with urinary iodine level of $100-499 \mu \mathrm{g} / \mathrm{L}$, and the difference is statistically significant $(\mathrm{P}<0.05)[20]$. It suggests that increased urine iodine is a risk factor for the increased incidence of GD. Reported that the prevalence of Graves' disease among residents in coastal areas of Shandong is $1.38 \%$, which is regionally distributed. The closer the residents are to the sea, the more iodine-containing food intake and the higher the urinary iodine excretion rate. The higher the morbidity rate, 30-39 years old is the most common age. When the urine iodine exceeds $300 \mu \mathrm{g} / \mathrm{L}$, the prevalence of Graves' disease increases with the increase of urine iodine [21]. Found that the MUI (134.17) of patients with hyperthyroidism in the Inner Mongolia Autonomous Region is significantly higher than that of patients with subclinical hyperthyroidism (103.21) [22]. Reported that the MUI $(305.50 \mu \mathrm{g} / \mathrm{L})$ of $102 \mathrm{GD}$ patients in the coastal area of western Guangdong is significantly higher than the control group $(263.51 \mu \mathrm{g} / \mathrm{L}, \mathrm{P}=0.011)$ [23]. Performed Kruskal-Wallis $\mathrm{H}$ test on urine iodine in GD and HT patients and found that their MUI was $302.95 \mu \mathrm{g} / \mathrm{L}$ and $354.4 \mu \mathrm{g} / \mathrm{L}$, respectively, which are higher than the control group $(257 \mu \mathrm{g} / \mathrm{L})$, and the difference is statistically significant. Significance $(\mathrm{P}<0.001)$, and the urine iodine level of the HT group is higher than the GD group, but the difference is not statistically significant $(\mathrm{P}=0.2078)$ [24]. The urinary iodine level of GD and HT patients is higher than the control group, which indicates that there is high iodine nutritional status in AITD patients, which may be one of the reasons for the development of AITD. The results of this study show that the constituent ratios of GD patients with different urine iodine concentrations are iodine adequate, iodine beyond, iodine excess and iodine deficiency from high to low. The sum of iodine beyond and iodine excess group is consistent with the iodine adequate group and is significantly higher than the iodine deficiency group, and the MUI of the GD-A group is significantly higher than that of the GD-B group, which indicates that high MUI is likely to cause the occurrence of GD, which is basically consistent with the above-mentioned research results. Epidemiological investigations have shown that IIH is usually related to the nutritional status of iodine before iodine supplementation. It mainly occurs in people who live in areas with high water iodine for a long time, people who have increased iodine intake in the short term after iodine supplementation is implemented in iodine-deficient areas, and women and elderly patients over 40 who receive drugs or contrast media containing large amounts of iodine. [25]. In short, the incidence of hyperthyroidism increases after iodine deficiency and normal iodine supplementation. With the increase in iodine supplementation speed and iodine supplementation dosage, the incidence of hyperthyroidism also increases. Nutritional status and iodine supplementation are closely related to the increase in the incidence of hyperthyroidism. Therefore, regular monitoring of urine iodine and timely adjustment of the iodized salt concentration can ensure the control of IDD and prevent the occurrence of IID. The possible pathogenesis of IIH is mainly related to long-term excessive iodine intake. With the removal of the Wolff-Chaik off effect, the thyroid can still synthesize a large amount of $\mathrm{TH}$, and it is retained in the thyroid follicles; it induces mutations in the TSH receptor gene, causing TSH-cAMP information. The conduction pathway is continuously activated and is related to the formation of autonomous nodules. However, there are also GD patients with urinary iodine content $(\mu \mathrm{g} / \mathrm{L})$ (141.16 \pm 92.79$)$ significantly lower than the control group (202.2 \pm 39.48$)$. Graves' disease is mainly characterized by iodine deficiency, followed by sufficient iodine and high iodine [26], and there is no statistically significant difference in the cumulative incidence of hyperthyroidism in areas with mild iodine deficiency, iodine excess, and iodine excess [27], and MUI is within the range of $100-600 \mu \mathrm{g} / \mathrm{L}$ iodine intake, which increases with iodine intake. It is reported that the prevalence of diffuse gonorrhea is gradually decreasing [28]. The difference of poor results may be related to the study 
object, regional distribution and differences in iodine levels. It shows that chronic iodine overdose does not significantly increase the risk of autoimmune hyperthyroidism. After the implementation of USI in areas with mild iodine deficiency, it does not increase the incidence of hyperthyroidism, suggesting that excessive iodine intake may not cause autoimmunity in the environment Risk factors for the onset of hyperthyroidism. At present, the international thyroid academia and endemic academia generally believe that the increase in the incidence of hyperthyroidism is inevitable in the process of iodine supplementation to prevent IDD. Many countries have experienced a similar process. At present, the dose and speed of iodine supplementation, as well as the relationship between the degree of iodine deficiency and the occurrence of hyperthyroidism are still unclear, and it is also very difficult to divide the susceptible population [29]. Therefore, the exact relationship between urinary iodine content and the onset of GD and its mechanism need to be conducted with a large sample of prospective studies.

The effect of iodine on the thyroid hormones of GD patients has attracted clinical attention. Different iodine nutritional status can affect the level of thyroid hormone andits autoantibodies. That 252 elderly patients with thyroid disease have statistical significance in TSH and FT3 in the iodine deficiency group and the iodine sufficient or over-adequate group, and there were statistics in the iodine excess group and the iodine sufficient or over-adequate group. The content of FT4 is not statistically significant among the three groups. It is suggested that excessive or insufficient intake of iodine in the elderly can lead to an increase in thyroid diseases. Adequate or excessive intake of iodine is very important to maintain normal thyroid function in the elderly [30]. The iodine nutritional status and the relationship between different urine iodine levels and thyroid diseases with 2650 physical examination populations in Inner Mongolia Autonomous Region. The results show that urine iodine levels are positively correlated with FT4 and FT3, and FT4 is correlated with TSH and TGAb [22]. It is negatively correlated, positively correlated with FT3, and TPOAb is positively correlated with TGAb. hat the serum FT3 and FT4 levels of women in high-iodine areas are significantly lower than those in iodine-adapted areas, while TSH levels are significantly increasing. The abnormal thyroid function rate and TSH levels of autoantibody-positive patients are significantly higher than those in iodine-adequate areas. In addition, excessive iodine intake and positive thyroid autoantibodies can increase the potential risk of disease [31]. Taked a cross-sectional random sample of 1995 healthy people in Urumqi to investigate the correlation between thyroid disease and urine iodine. It finds that there is no statistically significant difference in urinary iodine levels among the normal and abnormal thyroid function groups, and the positive and negative antibody groups $(\mathrm{P}>0.05)$ [32]. There is no obvious correlation between urinary iodine and thyroid function and autoantibodies, which suggests that urinary iodine level does not affect thyroid function and autoantibody expression, but no further analysis of iodine deficiency, iodine excess and most people with adequate iodine. The relationship between iodine nutritional status and thyroid function and autoantibodies. That the MUI of GD patients in the coastal areas of western Guangdong is more than $300 \mu \mathrm{g} / \mathrm{L}$, the median of the two autoantibodies is higher than the other groups, and the differences in urinary iodine levels among the four groups are statistically significant. ( $\mathrm{P}=0.023$ and $\mathrm{P}=0.038$ ), but there are no statistically significant differences among FT3, FT4, and TSH groups. It suggests that the adult iodine intake in this area is too high. Iodine may induce the occurrence of GD and aggravate GD exophthalmos [23]. That thyroid stimulating hormone receptor antibody (TRAb) is positively correlated with FT4, FT3, and thyroid volume in patients with GD hyperthyroidism. In the GD group, the positive rate of TRAb is significantly higher than that of other hyperthyroidism groups, while the positive rates of TPOAb and TGAb are lower than those of other hyperthyroidism groups $(\mathrm{P}<0.01)$ [33]. The two groups of patients have increased FT3 and FT4 and decreased TSH. The difference is not statistically significant $(\mathrm{P}>0.05)$. It is suggested that high iodine intake aggravates the autoimmunity of GD patients, and the iodine intake of GD patients should be limited. The results of this study show that the serum TSH levels of the GD group, the GD-A group and the GD-B group are significantly lower than that of the $\mathrm{NC}(\mathrm{P}<0.05)$, and the difference between the GD-A and GD-B groups is statistically significant $(\mathrm{P}<0.05)$. With the hyperthyroidism of GD-B and GD-A, the TSH level gradually decreases $(\mathrm{P}<0.05)$. The levels of serum FT3 and FT4 in GD, GD-A and GD-B groups are higher than those in $\mathrm{NC}$, but there is no significant difference in FT3 and FT4 levels between GD-B group and NC group $(\mathrm{P}>0.05)$. There is no significant difference in serum $\mathrm{T} 3$ and T4 levels among the GD, GD-A and GD-B groups and the NC group $(\mathrm{P}>0.05)$. This is consistent with the results of some of the above studies, indicating that the effect of high iodine intake on TSH of GD patients is significantly better than that of FT3 and FT4, but has no effect on T3 and T4 levels. It needs to be further verified by large sample studies.

Iodine can affect the level of thyroid autoantibodies in GD patients. Logistic multiple stepwise regression analysis finds that the occurrence of GD is positively correlated with the log values of TPOAb and urine iodine. TPOAb and TGAb in patients with coastal Graves' disease are higher than those in the inland control group $(\mathrm{P}<0.05)[21]$. It is proposed that in order to prevent the occurrence of Graves' disease, urine iodine should be controlled within $300 \mu \mathrm{g} / \mathrm{L}$. That in iodine overdosing and iodine overdosing communities with TPOAb positive, TSH increases significantly more than in mild iodine deficient communities, suggesting that increasing iodine intake can cause abnormal thyroid function in people with thyroid autoimmune abnormalities, The risk of increased [34]. That the positive rate of TPOAb in GD patients is significantly higher than that of controls, and the percentage of both TPOAb and TGAb antibodies simultaneously positive in GD patients is significantly higher than in asymptomatic patients [35]. That the positive rate of TPOAb and TGAb in the GDI group higher than MUI $(84.2 \%, 78.9 \%$, respectively) is significantly higher than the antibody and lower than the 
MUIGDII group $(62.5 \%, 50.0 \%)$ (P Respectively $<0.05$, $\mathrm{P}<0.01$ ), and the percentages of TPOAb and TGAb high titer group in GDI patients are higher than those in GDII group $(\mathrm{P}<0.05)$ [4]. Stratify the thyroid function of 1,098 inpatients in the Department of Endocrinology, People's Hospital of Xinjiang Uygur Autonomous Region, and find that the positive rate of autoantibodies in the 221 clinical thyroid dysfunction group is higher than the 316 subclinical thyroid dysfunction group. But the difference is not statistically significant $(\mathrm{P}>0.05)$, and the positive rate of antibodies in each abnormal thyroid function group is significantly higher than the normal thyroid function group $(\mathrm{P}<0.01)$ [36]. Stratify according to antibody titers and find that patients with high-titer TPOAb GD have a high proportion of three symptoms at the same time, and whether TGAb is at a lower or higher level, patients have thyrotoxicosis, goiter, and eye disease. There is no significant difference in the ratio. Two GD patients with different antibody titers have no statistically significant differences in FT3 and FT4, suggesting that there may be no clear correlation between the degree of abnormal thyroid function and the degree of thyroid autoimmunity, and abnormal thyroid function The manifestation is affected by the characteristics and degree of autoimmunity, especially closely related to TPOAb. The higher the level, the more diverse clinical manifestations. Stratified by TPOAb level, the clinical manifestations of different groups are different [37]. That the levels of FT3, FT4, TGAb and TPOAb in GD patients are significantly higher than those in the control group $(\mathrm{P}<0.01)$, TSH is lower than the control group, and the TPOAb titer is lower than that in the HT group, and the difference is statistically significant $(\mathrm{P}<0.01)$ (move up) [38]. That TPOAb $(232.47 \pm 17.82 \mathrm{U} / \mathrm{mL})$ of GD patients in the high-activity $\mathrm{TRAb}$ group is significantly higher than that in the medium-activity group $(190.05 \pm 32.87 \mathrm{U} / \mathrm{mL})$ and the low-activity group $(182.51 \pm 26.19 \mathrm{U} / \mathrm{mL}))$, the difference is statistically significant (all $\mathrm{P}<0.01$ ) [39]. The high activity group FT3 and FT4 are significantly higher than the medium activity group and the low activity group, and the difference is statistically significant (all $\mathrm{P}<0.01$ ). The results of this study show that serum TPOAb and TGAb levels in GD, GD-A and GD-B groups are significantly higher than $\mathrm{NC}(\mathrm{P}<0.01)$, regardless of GD-A or GD-B group, TPOAb and TGAb levels Both are significantly increased, and the serum TPOAb and TGAb levels in the GD-A group are also higher than those in the GD-B group. That is, the higher the urine iodine level, the higher the thyroid autoantibody level, and the more severe the damage to the thyroid function of GD patients. This is consistent with the results of most of the above studies, and also verify the Jin Ying's group [20] that the percentage of TPOAb, TPOAb and $\mathrm{TGAb}$ simultaneous positive in the GD group is significantly higher than that in the subclinical hyperthyroidism group $(\mathrm{P}<0.05)$, and the latter is also significantly higher. In the normal thyroid function group $(\mathrm{P}<0.05)$, the positive rate of TGAb in the GD group and the subclinical hyperthyroidism group is significantly higher than that in the normal thyroid function group $(\mathrm{P}<0.01)$, that is, the two antibodies of TPOAb and TGAb are single or both positive. The GD group has the highest percentage, followed by the subclinical hyperthyroidism group, and the normal thyroid function group has the lowest results. It is suggested that the level and positive rate of thyroid autoantibodies are related to the degree of hyperthyroidism and the condition of GD. However, the results of serum TPOAb and TGAb levels of healthy people in Guiyang City in areas with excess iodine excess are higher than those of iodine deficiency and excess iodine $(\mathrm{P}<0.05)$ [40]. It may be related to the difference in the research subjects, gender, age, and thyroid disease composition, and further research is needed. That there is no significant difference in the incidence of hyperthyroidism in iodine deficiency, iodine sufficient and iodine overdose areas, but the positive rates of TPOAb and TGAb in subclinical hyperthyroidism patients in iodine overdose areas are higher than the other two regions. TPOAb positive, especially those with normal thyroid function and subclinical hyperthyroidism who are both TPOAb and TGAb antibodies at the same time may be more likely to develop clinical GD [41]. However, the mechanism of TPOAb and TGAb in the occurrence of GD is still unclear.

In short, the level of iodine nutrition is essential to maintain the body's thyroid function and its autoantibody levels. Too much or too little iodine intake will directly affect the physiological processes of iodine uptake, transport, oxidation, synthesis and secretion of thyroid hormone, and ultimately lead to the occurrence of thyroid diseases such as GD. With the implementation of the USI policy, residents' iodine deficiency has been effectively prevented and controlled, but with that, the prevalence of thyroid diseases and the changes in the spectrum of thyroid diseases have gradually attracted people's attention. The effect of different iodine nutritional status on the thyroid function stratification and autoantibodies of GD patients will provide scientific basis for the precise treatment and prevention of GD. The mutual influence of iodine and other environmental factors, genetic and immune factors on GD and its mechanism of action remain to be $\mathrm{Yu}$ in-depth study. It is imperative to increase the detection of urinary iodine levels in different populations, to carry out monitoring and evaluation of thyroid function, and to adjust and establish scientific methods of iodine supplementation suitable for the national conditions of our country.

\section{Conclusion}

Composition ratio of GD patients with different urine iodine concentration are adequate iodine, iodine beyond, iodine excess and iodine deficiency from high to low. The sum of iodine beyond and iodine excess group is consistent with the proportion of iodine adequate group, which is significantly higher than iodine. The lack group, and the MUI of the GD-A group is significantly higher than the GD-B group, which indicates that high MUI is likely to cause the occurrence of GD. The serum TSH level of GD patients is significantly lower than $\mathrm{NC}$ (both $\mathrm{P}<0.05$ ). The difference between GD-A and GD-B is statistically significant $(\mathrm{P}<0.05)$. GD-B and GD-A increase with hyperthyroidism, and TSH level gradually decreases $(\mathrm{P}<0.05)$. The serum FT3 and FT4 levels of GD patients are higher than NC, while the GD-B group FT3 
and FT4 levels and the GD, GD-A and GD-B serum T3 and T4 levels are not significantly different from the $\mathrm{NC}$ group $(\mathrm{P}>0.05)$. It indicates that the effect of high iodine intake on TSH of GD patients is significantly better than FT3 and FT4, but has no effect on T3 and T4. Serum TPOAb and TGAb levels in GD patients are significantly higher than $\mathrm{NC}$ $(\mathrm{P}<0.01)$, and the GD-A group is also higher than the GD-B group. The effect of high-level antibodies on thyroid hormone is significantly better than low-level antibodies influences. That is, the higher the urine iodine level, the more obvious the change of thyroid hormone, and the higher the level of auto-antibodies, the more severe the damage to thyroid function. Therefore, regular monitoring of urinary iodine and thyroid hormone levels in patients with GD is of great significance for the precise prevention and treatment of GD.

\section{Acknowledgements}

Cangzhou Key R \& D Plan and Guidance Project (Project No: 1833020011).

Natural Science Foundation of Cangzhou Medical College (No. 18Z015).

\section{References}

[1] Wang L, Wang B, Chen SR, et al. Effect of Selenium Supplementation on Recurrent Hyperthyroidism Caused by Graves' Disease: A Prospective Pilot Study [J]. HormMetab Res, 2016, 48 (9): 559-564.

[2] Burch HB, Cooper DS. Management of Graves'disease: A Review [J]. JAMA, 2015, 314 (23): 2544-2554.

[3] Li HX, Xiang N, Hu WK, et al. Relation between therapy options for Graves' disease and the course of Graves' ophthalmopathy: a systematic review and meta-analysis [J]. J Endocrinol Invest, 2016, 39 (11): 1225-1233.

[4] Su JP, Su SO, Zhang B, et al. The effects of different iodine intake on autoimmune of Graves'disease [J]. Clin Med China, 2012, 28 (1): 44-46.

[5] San ZY. Guide to diagnosis and Treatment of Thyroid diseases in China [J]. Chin J Prac Inter Med, 2008, 28 (4): 260-261.

[6] Chinese Medical Association Endocrine Credits "China thyroid disease treatment guidelines" Writing Group. China thyroid disease treatment guidelines-iodine deficiencydisorders [J]. Journal of Internal Medicine, 2008, 47 (8): 689-690.

[7] Zhang YP, Yan YQ, Liu L J, et al. Urine ammonium persulfate digestion with a low arsenic content arsenic cerium catalytic spectrophotometric method [J]. Chin J Endemiol, 2013, 32 (1): 95-100.

[8] Wu ZF, LiangY, Chen XQ. Investigation of urinary iodine in patients with thyroid disease [J]. You Jiang Med, 2010, 38 (2): 188-189.

[9] Huang SY, Yan YM, Wu M, et al. Analysis of Urinary Iodine Content of Newly Diagnosed Thyroid Disease Patients in Xiaogan [J]. Guangzhou Microelement Science 2015, 22 (1): 24-27.
[10] Wang FL, Hou ZJ, Liu YZ, et al. Analysis of iodine nutritional status in patients with abnormal thyroid function [J]. China Medi Herald, 2018, 15 (25): 64-67.

[11] Laurberg P, Cerqueira C, Ovesen L, et al. Iodine intake as a determinant of thyroid disorders in populations [J]. Best Pract Res Clin Endocrinol Metab, 2010, 24 (1): 13-27.

[12] Tan X, Cao XX, He L. Effect of iodine excess on thyroid function and its pathogenesis of diseases [J]. Chin J Ctrl Endem Dis, 2019, 34 (1): 35-37.

[13] Li K, Yu FC, Kang WM. Iodine intake and thyroid disease [J]. Chin J Endem, 2016, 35 (3): 166-169.

[14] Zhang KZ, Lin YC, Fang ZP, et al. The effect of salt iodization for 10 years on the prevalences of endemic goiter and hyperthyroidism [J]. Chin J EndocrinolMetab, 2002, 18 (5): 342-344.

[15] Hou X, Wang J, QQ, et al. Epidemiologic comparative study of hyperthyroidism beforeand after salt iodization in different iodine intake regions of Jilin province $[\mathrm{J}]$. Chin $\mathrm{J}$ Endocrinol Meta, 2003, 19 (2): 104-105.

[16] Fan X, Chen SK. Relationship of Io dine Excessiveness an d T hyroid Function [J]. Med Reca, 2011, 17 (14): 2165-2167.

[17] Mostbeck A, Galvan G, Bauer P, et al. The incidence of hyperthyroidism in Austria from 1987 to 1995 before and after an increase in salt iodization in 1990. Eur J Nucl Med, 1998, 25: 367-374.

[18] Li CQ, Yin HQ, Zhang CK, et al. Epidemiological investigation of Graves's disease in one hundred thousand people in Daqingarea [J]. National Medical Journal of China, 1996, 76 (6): 443-446.

[19] Shan Z, Chen L, Lian X, et al. Iodine status and prevalence of thyroid disorders after introduction of mandatory universal salt iodization for 16 years in China: a crosssectional study in 10 cities [J]. Thyroid 2016, 26 (8): 1125-1130.

[20] JinY, Teng WP, Yuan B, et al. Thyroid autoimmunity in members from multiplex families with Graves' disease and effect of iodine intake on its incidence [J] Chin J Endocrinol Metab, 2001, 17 (2): 79-82.

[21] Yan SL, Wang YG, Wang F, et al. Relationship between iodine in urine and Graves's disease along coastal district in shading [J]. Chin J Endem, 2004, 23 (3): 245-247.

[22] Na BQ, Zhang XL, Wang SY, et al. Analysis of thyroid function under different iodine nutritional status $[\mathrm{J}]$. Chin $\mathrm{J}$ Endem, 2019, 38 (3): 235-238.

[23] Chen XM, Guo ZQ, Zhou F, et al. Correlation of concentrations of iodine in urine with Graves disease along coastal area of western Guangdong [J]. China Tropi Med, 2010, 10 (8): 922-923.

[24] Cong JN, Zhao YJ, Li G, et al. The changes of SOD and MDA in serum of patients with autoimmune thyroid disease and the relationship between SOD and urine iodine Guangzhou Microelement Science, 2011, 18 (8): 12-15.

[25] Teng W, Shan Z, Teng X, et al, Effect of iodine intake on thyroid disea-ses in China [J]. N Engl J Med, 2006, 354 (26): 2783-2793. 
[26] Huang WT, Huang W, Chang ZY, et al. Sundy on the relations between Graves disease (GD) and urine iodine in Guishinan area [J]. Chin J Ctrl Endem, 2004, 19 (2): 117-119.

[27] Yang F, Shan ZY, Teng XC, et al. Chronic iodine excess does not increase the incidence of hyperthyroidism: a prospective community-based epidemiological survey in China $[\mathrm{J}]$. Eur J Endocrinol. 2007, 156 (4): 403-408.

[28] Hu FN, Teng XC, Teng WP, et al. A comparative epidemic study of goiter and thyroid nodules in areas with different iodine intake [J]. Chin J Endem, 2002, 21 (6): 464-467.

[29] Teng WP. Common salt iodization and hyperthyroidism [J]. Chin J Endocrinol Metab 2000, 16 (3): 137-138.

[30] Wang YJ, ZhouW, Yang CX. Relationship between iodine nutritional status and thyroid disease in the elderly [J]. Cardiovascular Dise J integtradil Chin West Med, 2018, B6 (5): 98-99.

[31] Ren YT, Jia QZ, Zhang XD, et al. Epidemiological in vestigation on thyroid disease a mongfertile women in diferent iodine in take areas of Shanxi province [J]. Chin J Epidemiol, 2014, 35 (1): 45-48.

[32] Wang XL, OsimanReziwan, Ma FH, et al. Changes of thyroid function, thyroid antibodies and urinary iodine among permanent residents of Urumqi in Xinjiang $[\mathrm{J}]$. Chin $\mathrm{J}$ Epidemiol, 2015, 36 (8): 811-814.

[33] Xu YH, Cheng DX, Jin YS, et al. The significance of iodine intake and TRAb in the pathogenesis and clinical outcome of Graves disease [J]. Chia Mod Med, 2016, 23 (28): 75-77.
[34] Li YS, Jin Y, Teng WP, et al. The Comparative Screening for Thyroid Autoantibodies in Areas with Different Iodine Intakes [J]. Shanghai J Immun 2002, 22 (2): 91-95.

[35] Burek CL, Hoffman WH, Rose NR. The presence of thyroid autoantibodies in children and adolescents with autoimmune thyroid disease and in their siblings and parents [J]. ClinImmunol Immunopathol, 1982, 25 (3): 395-404.

[36] Song XX, Guo YY, Ma XQ. Analysis of thyroid function and the iodine nutrition status of Uygur and Han hospitalized patients in Xinjiang [J]. Hainan Med J, 2016, 27 (24): 3994-3997.

[37] Chen XY, Zhang ZT, Deng SY, et al. Clinical manifestation of Graves disease patients with different levels of thyroid related autoantibodies [J]. New Med, 2011, 42 (12): 789-792.

[38] Wang B, Meng JH. Research of thyroid autoantibodies in the diagnosis of Graves's disease and Hashimoto's thyroiditis s [J]. Chin J Lab Diagn. 2018, 22 (2): 207-209.

[39] Wang YL, Yao PW, Zhou LL, et al. Recurrence of Hyperthyroidism in Patients with Graves'sdisease and Elevated Thyrotropin Receptor Antibody Levels [J]. Acta Med UnivSci Tech Hua zhong, 2016, 45 (1): 95-97

[40] Qin C, Zhang Q. Influence of Iodine Nutrient Status on Autoimmune Thyroid Disease in Iodine Super Excessive Area [J]. J Guiyang Med Coll, 2016, 41 (2): 194-196.

[41] Yang F, Teng WP, Shan ZY, et al. A comparative epidemiologic survey of hyperthyroidism in areas with different iodine intake [J]. Chin J Endocrinol Metab, 2001, 17 (4): 197-201. 